International Mathematical Forum, Vol. 8, 2013, no. 37, 1797 - 1812

HIKARI Ltd, www.m-hikari.com

http://dx.doi.org/10.12988/imf.2013.310190

\title{
Some Results of Weighted Norlund-Euler
}

\section{Statistical Convergence}

\author{
${ }^{1}$ Ekrem A. Aljimi \\ Department of Mathematics, University of Tirana, Tiranë, Albania \\ ekremhalimii@yahoo.co.uk \\ ${ }^{2}$ Elida Hoxha \\ Department of Mathematics, University of Tirana, Tiranë, Albania \\ hoxhaelida@yahoo.com \\ ${ }^{3}$ Valdete Loku \\ Department of Computer Sciences and Applied Mathematics \\ College, Vizioni per Arsim, Ahmet Kaciku, Nr=3, Ferizaj, 70000, Kosova \\ Copyright (C) 2013 Ekrem A. Aljimi, Elida Hoxha and Valdete Loku. This is an open access \\ article distributed under the Creative Commons Attribution License, which permits unrestricted \\ use, distribution, and reproduction in any medium, provided the original work is properly cited.
}

\begin{abstract}
In this paper we will define the new weighted statistically summbaility method, known as the weighted Norlund-Euler statistical convergence. We will show some properties of this method and we have proved Korovkin type theorem.
\end{abstract}

\section{Mathematics Subject Classification: 40G15}

Keywords: Norlund-Euler type means, weighted Norlund-Euler statistical convergence, sequence spaces, summability 


\section{INTRODUCTION}

The idea of statistical convergence which is closely related to the concept of natural density or asymptotic density of a subset of the set of natural numbers $N$, was first introduced by Fast [3]. The weighted statistical convergence is defined by V. Karakaja and T.A.Chishti [2]. And the well defined version of the statistically weighted convergence sequences is given by Mursaleen et al., [15]. The interesting role in our results play the product of summability methods $(N, p, q)(E, 1)$ and we call it Norlund-Euler summability method. The concept of statistical convergence plays an important role in the summability theory and functional analysis. The relationship between the summability theory and statistical convergence has been introduced by Schoenberg [7]. Afterwards, the statistical convergence has been studied as a summability method by many researchers such as Fridy [8], Freedman et al. [9], Kolk [10, 11], Fridy and Miller [12], Fridy and Orhan [13,14], Mursaleen et al., [15], Savaş [16], Braha [3-5]. Also, some topological properties of statistical convergence sequence spaces have been studied by Salat [17]. Besides in [18, 19], Connor showed the relations between statistical convergence and functional analysis.

In general, statistical convergence of weighted means is studied as a class of regular matrix transformations. In this work, we introduce and study the concept of weighted Norlund-Euler statistical convergence. The relations among $(N, p, q)(E, 1)$-summability and $S_{(N E)}$ sequence of weighted statistically convergent sequences related to the weight $(N, p, q)(E, 1)$.

Let $K \subseteq N$ and $K_{n}=\{k \in K: k \leq n\}$. Then the natural density of $K$ is defined by $\delta(K)=\lim _{n \rightarrow \infty} \frac{\left|K_{n}\right|}{n}$ if the limit exists, where $\left|K_{n}\right|$ denotes the cardinality of $K_{n}$.

A sequence $x=\left(x_{k}\right)$ of real numbers is said to be statistically convergent to provided that for every $\varepsilon>0$ the set $K(\varepsilon)=\left\{k \in K:\left|x_{n}-L\right| \geq \varepsilon\right\}$ has natural density zero (Fast [5] and Steinhauss [14]), for each $\varepsilon>0$,

$\lim _{n \rightarrow \infty} \frac{1}{n}\left|\left\{j \leq n:\left|x_{j}-L\right| \geq \varepsilon\right\}\right|=0$ 
in this case we write $L=s t-\lim x$ or $(\mathrm{S}-\lim \mathrm{x}=\mathrm{L})$. The symbol S denotes the set of all statistically convergent sequences.

Let $\sum_{n=0}^{\infty} x_{n}$ be a given infinite series with sequence of its $n^{\text {th }}$ partial sum $\left\{S_{n}\right\}$.

If $(E, 1)$ transform is defined as

$E_{n}^{1}=\frac{1}{2^{n}} \sum_{k=0}^{n}\left(\begin{array}{l}n \\ k\end{array}\right) S_{k}$

And we say that this summability method is convergent if $E_{n}^{1} \rightarrow S$ as $n \rightarrow \infty$. In this case we say the series $\sum_{n=0}^{\infty} x_{n}$ is $(E, 1)$ - summable to a definite number S. (Hardy [19])

And we will write $S_{n} \rightarrow S(E, 1)$, as $n \rightarrow \infty$.

Let $\left(p_{n}\right)$ and $\left(q_{n}\right)$ be the two sequences of non-zero real constants such that $P_{n}=p_{0}+p_{1}+\ldots+p_{n}, P_{-1}=p_{-1}=0$

$Q_{n}=q_{0}+q_{1}+\ldots+q_{n}, Q_{-1}=q_{-1}=0$

For the given sequences $\left(p_{n}\right)$ and $\left(q_{n}\right)$, convolution $p * q$ is defined by:

$R_{n}=p * q=\sum_{k=0}^{n} p_{n} q_{n-k}$

The series $\sum_{n=0}^{\infty} x_{n}$ or the sequence $\left\{S_{n}\right\}$ is summable to $S$ by generalized Norlund method and it is denoted by $S_{n} \rightarrow S(N, p, q)$ if

$t_{n}^{p, q}=\frac{1}{R_{n}} \sum_{v=0}^{n} p_{n-v} q_{v} S_{v}$

tends to S, as $n \rightarrow \infty$. 
Let us use in consideration the following method of summability:

$t_{n}^{p, q, E}=\frac{1}{R_{n}} \sum_{k=0}^{n} p_{n-k} q_{k} E_{k}^{1}=\frac{1}{R_{n}} \sum_{k=0}^{n} p_{n-k} q_{k} \frac{1}{2^{k}} \sum_{v=0}^{k}\left(\begin{array}{l}k \\ v\end{array}\right) S_{v}$.

If $t_{n}^{p, q, E} \rightarrow S$ as $n \rightarrow \infty$, then we say that the series $\sum_{n=0}^{\infty} x_{n}$ or the sequence $\left\{S_{n}\right\}$ is summable to $\mathrm{S}$ by Norlund-Euler method and it is denoted by $S_{n} \rightarrow S(N, p, q)(E, 1)$

Remark: If $p_{k}=1, q_{k}=1$, then we get Euler summability method

Let us denote by $|(N, p, q)(E, 1)|$ the sequence space all strongly convergent sequences $x=\left(x_{k}\right)$ which are $(N, p, q)(E, 1)$ summable to L:

$|(N, p, q)(E, 1)|=\left\{x=x_{n}: \lim _{n \rightarrow \infty} \frac{1}{R_{n}} \sum_{k=0}^{n} p_{n-k} q_{k} \frac{1}{2^{k}} \sum_{v=0}^{k}\left(\begin{array}{l}k \\ v\end{array}\right)\left|x_{v}-L\right|=0\right.$ for some $\left.L\right\}$

The matrix $A=\left(a_{n, k}\right)$ in $(N, p, q)(E, 1)$ - summability is given by

$a_{n, k}=\left\{\begin{array}{l}\frac{1}{R_{n}} p_{n-k} q_{k} \frac{1}{2^{k}} \sum_{v=0}^{k}\left(\begin{array}{l}k \\ v\end{array}\right), \text { if } k \leq n \\ 0, \text { if } k>n\end{array}\right.$

Now we are able to give the definition of the weighted statistical convergence related to the $(N, p, q)(E, 1)$ - summability method.

Definition 1.1. A sequence $x=\left(x_{k}\right)$ is said to be weighted Norlund-Euler statistical convergent if for every $\varepsilon>0$.

$\lim _{n \rightarrow \infty} \frac{1}{R_{n}}\left|\left\{k \leq R_{n}: p_{n-k} q_{k} \frac{1}{2^{k}} \sum_{v=0}^{k}\left(\begin{array}{l}k \\ n\end{array}\right)\left|x_{v}-L\right| \geq \varepsilon\right\}\right|=0$

The set of weighted Norlund-Euler statistical convergence sequence is denoted by $S_{N E}$ as follows: 
$S_{N E}=\left\{x=\left(x_{n}\right): \lim _{n \rightarrow \infty} \frac{1}{R_{n}}\left|\left\{k \leq R_{n}: p_{n-k} q_{k} \frac{1}{2^{k}} \sum_{v=0}^{k}\left(\begin{array}{l}k \\ n\end{array}\right)\left|x_{v}-L\right| \geq \varepsilon\right\}\right|=0\right.$, for some $\left.L\right\}$

If the sequence $x=\left(x_{k}\right)$ is $S_{N E}$-convergence, then we also use the notation $x_{k} \rightarrow L\left(S_{N E}\right)$.

Definition 1.2. A sequence $x=\left(x_{k}\right)$ is said to be $[(N, p, q)(E, 1)]_{r}-$ summable $(0<r<\infty)$

to the limit $L$ if $\lim _{n \rightarrow \infty} \frac{1}{R_{n}} \sum_{k=1}^{n} p_{n-k} q_{k} \frac{1}{2^{k}} \sum_{v=0}^{k}\left(\begin{array}{l}k \\ n\end{array}\right)\left|x_{v}-L\right|^{r}=0$

, and we write it as $x_{k} \rightarrow L\left([(N, p, q)(E, 1)]_{r}\right)$

In these case $L$ is called the limit $[(N, p, q)(E, 1)]_{r}-$ limit of $\boldsymbol{x}$.

In these paper, we establish the relation of $S_{N E}$ - convergence with statistical summability $(N, p, q)(E, 1)$ and $[(N, p, q)(E, 1)]_{r}$.

\section{MAIN RESULTS}

In our first theorem we establish the relation between $S_{N E}$-statistical convergence and statistical summability $(N, p, q)(E, 1)$.

Theorem 2.1. Let $p_{n-k} q_{k} \frac{1}{2^{k}} \sum_{v=0}^{k}\left(\begin{array}{l}k \\ v\end{array}\right)\left|x_{v}-L\right| \leq M$ for all $k \in N$. If a sequence $x=\left(x_{k}\right)$ is $S_{N E}$-statistically convergent to $L$ then it statistically summable $(N, p, q)(E, 1)$ to $L$ but not conversely.

Proof: Since $x=\left(x_{k}\right)$ is $S_{N E}$-statistically convergent to $L$, $\lim _{n \rightarrow \infty} \frac{1}{R_{n}}\left|\left\{k \leq R_{n}: p_{n-k} q_{k} \frac{1}{2^{k}} \sum_{v=0}^{k}\left(\begin{array}{l}k \\ v\end{array}\right)\left|x_{v}-L\right| \geq \varepsilon\right\}\right|=0$, 
where $K_{\varepsilon}=\left\{k \in N: p_{n-k} q_{k} \frac{1}{2^{k}} \sum_{v=0}^{k}\left(\begin{array}{l}k \\ v\end{array}\right)\left|x_{v}-L\right| \geq \varepsilon\right\}$.

Write $K_{R_{n}}(\varepsilon)=\left\{k \leq R_{n}: p_{n-k} q_{k} \frac{1}{2^{k}} \sum_{v=0}^{k}\left(\begin{array}{l}k \\ v\end{array}\right)\left|x_{v}-L\right| \geq \varepsilon\right\}$ and

$K_{R_{n}}^{c}(\varepsilon)=\left\{k \leq R_{n}: p_{n-k} q_{k} \frac{1}{2^{k}} \sum_{v=0}^{k}\left(\begin{array}{l}k \\ v\end{array}\right)\left|x_{v}-L\right|<\varepsilon\right\}$. Than

$\left|\mathrm{t}_{\mathrm{n}}^{\mathrm{p}, \mathrm{q}, \mathrm{E}}-L\right|=\left|\frac{1}{R_{n}} \sum_{k=0}^{n} p_{n-k} q_{k} \frac{1}{2^{k}} \sum_{v=0}^{k}\left(\begin{array}{l}k \\ v\end{array}\right) x_{v}-L\right|=\left|\frac{1}{R_{n}} \sum_{k=0}^{n} p_{n-k} q_{k} \frac{1}{2^{k}} \sum_{v=0}^{k}\left(\begin{array}{l}k \\ v\end{array}\right)\left(x_{v}-L\right)\right|$

$=\left|\frac{1}{R_{n}} \sum_{\substack{k=0 \\ k \in K_{R_{n}}(\varepsilon)}}^{n} p_{n-k} q_{k} \frac{1}{2^{k}} \sum_{\substack{v=0 \\ k \in K_{R_{n}}(\varepsilon)}}^{k}\left(\begin{array}{l}k \\ v\end{array}\right)\left(x_{v}-L\right)+\frac{1}{R_{n}} \sum_{\substack{k=0 \\ K_{R_{n}}^{c}(\varepsilon)}}^{n} p_{n-k} q_{k} \frac{1}{2^{k}} \sum_{\substack{v=0 \\ K_{R_{n}}^{c}(\varepsilon)}}^{k}\left(\begin{array}{l}k \\ v\end{array}\right)\left(x_{v}-L\right)\right|$

$\leq \frac{1}{R_{n}} \sum_{\substack{k=0 \\ K_{R_{n}}(\varepsilon)}}^{n} p_{n-k} q_{k} \frac{1}{2^{k}} \sum_{v=0}^{k}\left(\begin{array}{l}k \\ v\end{array}\right)\left|\left(x_{k}-L\right)\right|+\frac{1}{R_{n}} \sum_{\substack{k=0 \\ K_{R_{n}}^{c}(\varepsilon)}}^{n} p_{n-k} q_{k} \frac{1}{2^{k}} \sum_{v=0}^{k}\left(\begin{array}{l}k \\ v\end{array}\right)\left|\left(x_{k}-L\right)\right|$

$\leq \frac{1}{R_{n}} M\left|\left\{k \leq R_{n}: p_{n-k} q_{k} \frac{1}{2^{k}} \sum_{v=0}^{k}\left(\begin{array}{l}k \\ n\end{array}\right)\left|x_{v}-L\right| \geq \varepsilon\right\}\right|+\frac{1}{R_{n}} \sum_{k \in K_{R_{n}}^{c}(\varepsilon)} \varepsilon$

$=\frac{1}{R_{n}} M\left|\left\{k \leq R_{n}: p_{n-k} q_{k} \frac{1}{2^{k}} \sum_{v=0}^{k}\left(\begin{array}{l}k \\ n\end{array}\right)\left|x_{v}-L\right| \geq \varepsilon\right\}\right|+\varepsilon \frac{\left|K_{R_{n}}^{c}(\varepsilon)\right|}{R_{n}} \rightarrow 0+\varepsilon \cdot 1=\varepsilon$

as $n \rightarrow \infty$ which implies that $t_{n}^{p, q}=\frac{1}{R_{n}} \sum_{k=0}^{n} p_{n-k} q_{k} \frac{1}{2^{k}} \sum_{v=0}^{k}\left(\begin{array}{l}k \\ v\end{array}\right) x_{v} \rightarrow L$. That is, $x$ is $(N, p, q)(E, 1)$-summable to $L$ and hence statistically summable $(N, p, q)(E, 1)$ to $L$.

Example 1: Let us consider that $p_{n}=1$ and $q_{n}=1$ for all $n \in \mathrm{N}$. Also we define the following sequence:

$x=\left(x_{k}\right)$ as follows:

$x_{k}=\left\{\begin{array}{l}\sqrt{k}, \text { if } k=n^{2} \\ 0, \text { if } k \neq n\end{array}\right.$ 
Then we have $R_{n}=\sum_{k=0}^{n} 1=n+1$ and

$\frac{1}{R_{n}}\left|\left\{k \leq R_{n}: \sum_{k=0}^{n} p_{n-k} q_{k} \frac{1}{2^{k}} \sum_{v=0}^{k}\left(\begin{array}{l}k \\ v\end{array}\right)\left|x_{v}-0\right| \geq \varepsilon\right\}\right|=\frac{\sqrt{n}}{n+1} \rightarrow 0$, as $n \rightarrow \infty$.

On the other hand,

$\frac{1}{R_{n}} \sum_{k=0}^{n} p_{n-k} q_{k} \frac{1}{2^{k}} \sum_{v=0}^{k}\left(\begin{array}{l}k \\ v\end{array}\right)\left|x_{v}-0\right|=\frac{1}{n+1} \sum_{k=0}^{n} 1 \frac{1}{2^{k}} \sum_{v=0}^{k}\left(\begin{array}{l}k \\ v\end{array}\right) x_{v^{2}}=\frac{1}{n+1} \sum_{k=0}^{n} \frac{1}{2^{k}} \sum_{v=0}^{k}\left(\begin{array}{l}k \\ v\end{array}\right) v$

$=$

$\frac{1}{n+1} \sum_{k=0}^{n} \frac{k}{2}=\frac{n}{4} \rightarrow \infty$, as $n \rightarrow \infty$

These examples proves that converse is not true.

Next theorem gives the relation between $S_{N E}$-statistically convergent and $[(N, p, q)(E, 1)]_{r}$

\section{Theorem 2.2.}

a. Let a sequence $x=\left(x_{k}\right)$ is $[(N, p, q)(E, 1)]_{r}$ - summable to $L$. If the following are valid, then $x=\left(x_{k}\right)$ is $S_{N E}$-statistically convergent to $L$ :

Case 1: $0<r<1$ and $0 \leq\left|x_{k}-L\right|<1$

Case 2: $1 \leq r<\infty$ and $1 \leq\left|x_{k}-L\right|<\infty$.

b. Let $x=\left(x_{k}\right)$ is $S_{N E}$-statistically convergent to $L$ and $p_{n-k} q_{k} \frac{1}{2^{k}} \sum_{v=0}^{k}\left(\begin{array}{l}k \\ v\end{array}\right)\left|x_{v}-L\right| \leq M(\mathrm{k}=1,2,3, \ldots)$. If the following are valid, then $x_{k} \rightarrow L[(N, p, q)(E, 1)]_{r}:$

Case 1: $0<r<1$ and $1 \leq M<\infty$

Case 2: $1 \leq r<\infty$ and $0 \leq M<1$. 
Proof:

a. Since $p_{n-k} q_{k} \frac{1}{2^{k}} \sum_{v=0}^{k}\left(\begin{array}{l}k \\ v\end{array}\right)\left|x_{v}-L\right|^{r} \geq p_{n-k} q_{k} \frac{1}{2^{k}} \sum_{v=0}^{k}\left(\begin{array}{l}k \\ v\end{array}\right)\left|x_{v}-L\right|$

for case 1 . and case 2 , then as $n \rightarrow \infty$

$$
\begin{aligned}
& 0 \leftarrow \frac{1}{R_{n}} \sum_{k=0}^{n} p_{n-k} q_{k} \frac{1}{2^{k}} \sum_{v=0}^{k}\left(\begin{array}{l}
k \\
v
\end{array}\right)\left|x_{v}-L\right|^{r} \\
& \geq \frac{1}{R_{n}} \sum_{k=0}^{n} p_{n-k} q_{k} \frac{1}{2^{k}} \sum_{v=0}^{k}\left(\begin{array}{l}
k \\
v
\end{array}\right)\left|x_{v}-L\right| \geq \frac{1}{R_{n}} \sum_{\substack{k=0 \\
K_{R_{n}}(\varepsilon)}}^{n} p_{n-k} q_{k} \frac{1}{2^{k}} \sum_{\substack{v=0 \\
K_{R_{n}}(\varepsilon)}}^{k}\left(\begin{array}{l}
k \\
v
\end{array}\right)\left|x_{v}-L\right| \geq \\
& \frac{1}{R_{n}} \sum_{\substack{k=1 \\
K_{R_{n}}(\varepsilon)}}^{n} \varepsilon=\varepsilon \frac{\left|K_{R_{n}}(\varepsilon)\right|}{R_{n}} \\
& \lim _{n \rightarrow \infty} \frac{\left|K_{R_{n}}(\varepsilon)\right|}{R_{n}}=0 \text { where } \\
& K_{R_{n}}(\varepsilon)=\left\{k \leq R_{n}: p_{n-k} q_{k} \frac{1}{2^{k}} \sum_{v=0}^{k}\left(\begin{array}{l}
k \\
v
\end{array}\right)\left|x_{v}-L\right| \geq \varepsilon\right\}
\end{aligned}
$$

Hence $S_{N E}$-statistically convergent to $L$.

b. Suppose that $x=\left(x_{k}\right)$ is $S_{N E}$-statistically convergent to $L$. Then for $\varepsilon>0$, we have $\delta_{N E}\left(K_{\varepsilon}\right)=\delta_{N E}\left(\left\{k \in N: p_{n-k} q_{k} \frac{1}{2^{k}} \sum_{v=0}^{k}\left(\begin{array}{l}k \\ v\end{array}\right)\left|x_{v}-L\right| \geq \varepsilon\right\}\right)=0$. Since

$$
\begin{aligned}
& p_{n-k} q_{k} \frac{1}{2^{k}} \sum_{v=0}^{k}\left(\begin{array}{l}
k \\
v
\end{array}\right)\left|x_{v}-L\right| \leq M(\mathrm{k}=1,2, \ldots) \text {, we have } \\
& \frac{1}{R_{n}} \sum_{\substack{k=0 \\
K_{R_{n}}(\varepsilon)}}^{n} p_{n-k} q_{k} \frac{1}{2^{k}} \sum_{\substack{v=0 \\
K_{R_{n}}(\varepsilon)}}^{k}\left(\begin{array}{l}
k \\
v
\end{array}\right)\left|x_{v}-L\right|^{r}= \\
& \frac{1}{R_{n}} \sum_{\substack{k=0 \\
k \notin K_{R_{n}}(\varepsilon)}}^{n} p_{n-k} q_{k} \frac{1}{2^{k}} \sum_{\substack{v=0 \\
k \notin K_{R_{n}}(\varepsilon)}}^{k}\left(\begin{array}{l}
k \\
v
\end{array}\right)\left|x_{v}-L\right|^{r}+\frac{1}{R_{n}} \sum_{\substack{k=0 \\
k \in K_{R_{n}}(\varepsilon)}}^{n} p_{n-k} q_{k} \frac{1}{2^{k}} \sum_{\substack{v=0 \\
k \in K_{R_{n}}(\varepsilon)}}^{k}\left(\begin{array}{l}
k \\
v
\end{array}\right)\left|x_{v}-L\right|^{r}=S_{1}(n)+S_{2}(n)
\end{aligned}
$$

where 


$$
\begin{aligned}
& S_{1}(n)=\frac{1}{R_{n}} \sum_{\substack{k=0 \\
k \notin K_{R_{n}}(\varepsilon)}}^{n} p_{n-k} q_{k} \frac{1}{2^{k}} \sum_{\substack{v=0 \\
k \notin K_{R_{n}}(\varepsilon)}}^{k}\left(\begin{array}{l}
k \\
v
\end{array}\right)\left|x_{v}-L\right|^{r} \\
& S_{2}(n)=\frac{1}{R_{n}} \sum_{\substack{k=0 \\
k \in K_{R_{n}}(\varepsilon)}}^{n} p_{n-k} q_{k} \frac{1}{2^{k}} \sum_{\substack{v=0 \\
k \in K_{R_{n}}(\varepsilon)}}^{k}\left(\begin{array}{l}
k \\
v
\end{array}\right)\left|x_{v}-L\right|^{r} .
\end{aligned}
$$

Now if $k \notin K_{R_{n}}(\varepsilon)$ then $S_{1}(n)=\frac{1}{R_{n}} \sum_{\substack{k=0 \\ k \notin K_{R_{n}}(\varepsilon)}}^{n} p_{n-k} q_{k} \frac{1}{2^{k}} \sum_{\substack{v=0 \\ k \notin K_{R_{n}}(\varepsilon)}}^{k}\left(\begin{array}{l}k \\ v\end{array}\right)\left|x_{v}-L\right|^{r}$

$\leq \frac{1}{R_{n}} \sum_{\substack{k=0 \\ k \notin K_{R_{n}}(\varepsilon)}}^{n} p_{n-k} q_{k} \frac{1}{2^{k}} \sum_{\substack{=00 \\ k \notin K_{R_{n}}(\varepsilon)}}^{k}\left(\begin{array}{l}k \\ v\end{array}\right)\left|x_{v}-L\right|=\varepsilon \frac{1}{R_{n}}\left|K_{R_{n}}^{c}\right|=\varepsilon$

For

$$
k \in K_{R_{n}}(\varepsilon),
$$

we

have

$$
\begin{aligned}
& S_{2}(n)=\frac{1}{R_{n}} \sum_{\substack{k=0 \\
k \in K_{R_{n}}(\varepsilon)}}^{n} p_{n-k} q_{k} \frac{1}{2^{k}} \sum_{\substack{v=0 \\
k \in K_{R_{n}}(\varepsilon)}}^{k}\left(\begin{array}{l}
k \\
v
\end{array}\right)\left|x_{v}-L\right|^{r} \\
& \leq \frac{1}{R_{n}} \sum_{\substack{k=0 \\
k \in K_{R_{n}}(\varepsilon)}}^{n} p_{n-k} q_{k} \frac{1}{2^{k}} \sum_{\substack{v=0 \\
k \in K_{R_{n}}(\varepsilon)}}^{k}\left(\begin{array}{l}
k \\
v
\end{array}\right)\left|x_{v}-L\right| \leq\left(\sup _{\substack { k \\
\begin{subarray}{c}{k=0 \\
k \in K_{R_{n}}(\varepsilon){ k \\
\begin{subarray} { c } { k = 0 \\
k \in K _ { R _ { n } } ( \varepsilon ) } }\end{subarray}}^{n} p_{n-k} q_{k} \frac{1}{2^{k}} \sum_{\substack{v=0 \\
k \in K_{R_{n}}(\varepsilon)}}^{k}\left(\begin{array}{l}
k \\
v
\end{array}\right)\left|x_{v}-L\right|\right)\left(\frac{K_{R_{n}}(\varepsilon)}{R_{n}}\right) \\
& \leq M \frac{\left|K_{R_{n}}(\varepsilon)\right|}{R_{n}} \rightarrow 0, \text { as } n \rightarrow \infty \text {. Since } \\
& \delta_{N E}\left(K_{\varepsilon}\right)=\delta_{N E}\left(\left\{k \in N: p_{n-k} q_{k} \frac{1}{2^{k}} \sum_{v=0}^{k}\left(\begin{array}{l}
k \\
v
\end{array}\right)\left|x_{v}-L\right| \geq \varepsilon\right\}\right)=0 .
\end{aligned}
$$

Hence $x_{k} \rightarrow L[(N, p, q)(E, 1)]_{r}$.

\section{Application to approximation theorem}

Let $C[a, b]$ be the space of all functions $f$ continuous on $[a, b]$. We know that $C[a, b]$ is Banach space with norm 
$\|f\|_{\infty}=\sup _{x \in[a, b]}|f(x)|, f \in C[a, b]$

The classical Korovkin approximation theorem states as follows [26]:

Let $T_{n}$ be a sequence of positive linear operators from $C[a, b]$ into $C[a, b]$. Then $\lim _{n}\left\|T_{n}(f, x)-f(x)\right\|_{\infty}=0, \quad$ for all $f \in C[a, b]$ if only if $\lim _{n}\left\|T_{n}\left(f_{i}, x\right)-f_{i}(x)\right\|_{\infty}=0, \quad$ for $\quad i=0,1,2 \quad$ where $\quad f_{0}(x)=1, \quad f_{1}(x)=x$ dhe $f_{2}(x)=x^{2}$.

Its statistical version was given by Gadjiev and Orhan [25]. Such type of approximation theorems are proved by using the concept of almost convergence $[2],[6], \quad[21-27], \lambda$-statistical convergence $[29,31]$ and statistical lacunary summability [30]. Boyanov and Veselinov [23] have proved the Korovkin theorem on $C[0, \infty)$ by using the test functions $1, e^{-x}, e^{-2 x}$.In addition, some related papers on this topic can be found in [33-35]. In this paper, we generalize the result of Boyanov and Veselinov by using the notion of statistical summability $(N, p, q)(E, 1)$ and the same test functions $1, e^{-x}, e^{-2 x}$.We also give an example to justify that our result is stronger than that of Boyanov and Veselinov [23].

Let $C(I)$ be the Banach space with the uniform norm $\|,\|_{\infty}$ of all real-valued two dimensional continuous functions on $I=[0, \infty)$; provided that $\lim _{x \rightarrow \infty} f(x)$ is finite. Suppose that $L_{n}: C(I) \rightarrow C(I)$. We write $L_{n}(f ; x)$ for $L_{n}(f(s) ; x)$; and we say that $L$ is a positive operator if $L_{n}(f ; x) \geq 0$ for all $f(x) \geq 0$.

The following statistical version of Boyanov and Veselinov's result can be obtained as a special case of [24].

Teorem A. Let $\left(T_{k}\right)$ be a sequence of positive linear operators from $C(I)$ into $C(I)$. Than for all $f \in C(I)$.

$$
s t-\lim _{k \rightarrow \infty}\left\|T_{k}(f ; x)-f(x)\right\|_{\infty}=0
$$

if only if

$$
s t-\lim _{k \rightarrow \infty}\left\|T_{k}(1 ; x)-f(1)\right\|_{\infty}=0
$$




$$
\begin{aligned}
& s t-\lim _{k \rightarrow \infty}\left\|T_{k}\left(e^{-s} ; x\right)-e^{-x}\right\|_{\infty}=0 \\
& s t-\lim _{k \rightarrow \infty}\left\|T_{k}\left(e^{-2 s} ; x\right)-e^{-2 x}\right\|_{\infty}=0
\end{aligned}
$$

Similarly one can prove the $S_{N E}$-statistical version. Now we prove the following stronger version by using the notion of statistical summability $(N, p, q)(E, 1)$.

Teorem 3.1. Let $\left(T_{k}\right)$ be a sequence of positive linear operators from $C(I)$ into $C(I)$. Than for all $f \in C(I)$.

$$
N E(s t)-\lim _{k \rightarrow \infty}\left\|T_{k}(f ; x)-f(x)\right\|_{\infty}=0
$$

if only if

$$
\begin{aligned}
& N E(s t)-\lim _{k \rightarrow \infty}\left\|T_{k}(1 ; x)-f(1)\right\|_{\infty}=0 \\
& N E(s t)-\lim _{k \rightarrow \infty}\left\|T_{k}\left(e^{-s} ; x\right)-e^{-x}\right\|_{\infty}=0 \\
& N E(s t)-\lim _{k \rightarrow \infty}\left\|T_{k}\left(e^{-2 s} ; x\right)-e^{-2 x}\right\|_{\infty}=0
\end{aligned}
$$

Proof. Since each $1, e^{-x}, e^{-2 x}$ belongs to $C(I)$,conditions (3.1.1)-(3.1.3) follow immediately from (3.1.0). Let $f \in C(I)$. Then there exists a constant $M>0$ such that $|f(x)| \leq M$ for $x \in I$. Therefore,

$|f(s)-f(x)| \leq 2 M, \quad-\infty<s, x<+\infty$

It is easy to prove that for a given $\varepsilon>0$ there is a $\delta>0$ such that

$$
|f(s)-f(x)|<\varepsilon
$$

whenever $\left|e^{-s}-e^{-x}\right|<\delta$ for all $x \in I$.

Using (3.1.4) and (3.1.5), putting $\psi_{1}=\psi_{1}(s, x)=\left(e^{-s}-e^{-x}\right)^{2}$, we get 


$$
|f(s)-f(x)|<\varepsilon+\frac{2 M}{\delta^{2}}\left(\psi_{1}\right), \forall|s-x|<\delta .
$$

This is,

$$
-\varepsilon-\frac{2 M}{\delta^{2}}\left(\psi_{1}\right)<f(s)-f(x)<\varepsilon+\frac{2 M}{\delta^{2}}\left(\psi_{1}\right) .
$$

Now, operating $T_{k}(1 ; x)$ to this inequality since $T_{k}(f ; x)$ is monotone and linear. We obtain

$T_{k}(1 ; x)\left(-\varepsilon-\frac{2 M}{\delta^{2}}\left(\psi_{1}\right)\right)<T_{k}(1 ; x)(f(s)-f(x))<T_{k}(1 ; x)\left(\varepsilon+\frac{2 M}{\delta^{2}}\left(\psi_{1}\right)\right)$.

Note that $x$ is fixed and so $f(x)$ is constant number. Therefore,

$$
\begin{aligned}
& -\varepsilon T_{k}(1 ; x)-\frac{2 M}{\delta^{2}}\left(\psi_{1}\right) T_{k}(1 ; x)<(f(s)-f(x)) T_{k}(1 ; x)<\varepsilon T_{k}(1 ; x)+\frac{2 M}{\delta^{2}}\left(\psi_{1}\right) T_{k}(1 ; x) \\
& -\varepsilon T_{k}(1 ; x)-\frac{2 M}{\delta^{2}} T_{k}\left(\psi_{1} ; x\right)<T_{k}(f ; x)-f(x) T_{k}(1 ; x)<\varepsilon T_{k}(1 ; x)+\frac{2 M}{\delta^{2}} T_{k}\left(\psi_{1} ; x\right)
\end{aligned}
$$

Also,

$$
\begin{aligned}
& T_{k}(f ; x)-f(x)=T_{k}(f ; x)-f(x) T_{k}(1 ; x)+f(x) T_{k}(1 ; x)-f(x) \\
& =T_{k}(f ; x)-f(x) T_{k}(1 ; x)+f(x)\left[T_{k}(1 ; x)-1\right]
\end{aligned}
$$

If follows (3.1.6) and (3.1.7) that

$$
T_{k}(f ; x)-f(x)<\varepsilon T_{k}(1 ; x)+\frac{2 M}{\delta^{2}} T_{k}\left(\psi_{1} ; x\right)+f(x)\left[T_{k}(1 ; x)-1\right]
$$

Now

$$
\begin{aligned}
& T_{k}\left(\psi_{1} ; x\right)=T_{k}\left(\left(e^{-s}-e^{-x}\right)^{2} ; x\right)=T_{k}\left(e^{-2 s}-2 e^{-s} e^{-x}+e^{-2 x} ; x\right) \\
& =T_{k}\left(e^{-2 s} ; x\right)-2 e^{-x} T_{k}\left(e^{-s} ; x\right)+e^{-2 x} T_{k}(1 ; x) \\
& =\left[T_{k}\left(e^{-2 s} ; x\right)-e^{-2 x}\right]-2 e^{-x}\left[T_{k}\left(e^{-s} ; x\right)-e^{-x}\right]+e^{-2 x}\left[T_{k}(1 ; x)-1\right]
\end{aligned}
$$


Using (3.1.8), we obtain

$T_{k}(f ; x)-f(x)<\varepsilon T_{k}(1 ; x)+\frac{2 M}{\delta^{2}}\left\{\left[T_{k}\left(e^{-2 s} ; x\right)-e^{-2 x}\right]-2 e^{-x}\left[T_{k}\left(e^{-s} ; x\right)-e^{-x}\right]+e^{-2 x}\left[T_{k}(1 ; x)-1\right]\right\}+$

$+f(x)\left[T_{k}(1 ; x)-1\right]$

There for

$\left|T_{k}(f ; x)-f(x)\right| \leq \varepsilon+(\varepsilon+M)\left|T_{k}(1 ; x)-1\right|+\frac{2 M}{\delta^{2}}\left|e^{-2 x}\right| T_{k}(1 ; x)-1\left|+\frac{2 M}{\delta^{2}}\right| T_{k}\left(e^{-2 x} ; x\right)-e^{-2 x} \mid+$

$\frac{4 M}{\delta^{2}}\left|e^{-x}\right|\left|T_{k}\left(e^{-s} ; x\right)-e^{-x}\right| \leq \varepsilon+\left(\varepsilon+M+\frac{4 M}{\delta^{2}}\right)\left|T_{k}(1 ; x)-1\right|+\frac{2 M}{\delta^{2}}\left|e^{-2 x}\right| T_{k}(1 ; x)-1\left|+\frac{2 M}{\delta^{2}}\right| T_{k}\left(e^{-2 x} ; x\right)-e^{-2 x} \mid+$

$\frac{4 M}{\delta^{2}}\left|T_{k}\left(e^{-s} ; x\right)-e^{-x}\right|$.

Since $\left|e^{-x}\right| \leq 1$ for all $x \in I$. Now, taking $\sup _{x \in I}$, we get

$\left\|T_{k}(f ; x)-f(x)\right\|_{\infty} \leq \varepsilon+K\left(\left\|T_{k}(1 ; x)-1\right\|_{\infty}+\left\|T_{k}\left(e^{-s} ; x\right)-e^{-x}\right\|_{\infty}+\left\|T_{k}\left(e^{-2 s} ; x\right)-e^{-2 x}\right\|_{\infty}\right)$,

where $K=\max \left\{\varepsilon+M+\frac{4 M}{\delta^{2}}, \frac{2 M}{\delta^{2}}\right\}$. Hence

$\left\|T_{k}(f ; x)-f(x)\right\|_{\infty} \leq$

$\varepsilon+K\left(\left\|T_{k}(1 ; x)-1\right\|_{\infty}+\left\|T_{k}\left(e^{-s} ; x\right)-e^{-x}\right\|_{\infty}+\left\|T_{k}\left(e^{-2 s} ; x\right)-e^{-2 x}\right\|_{\infty}\right)$,

Now replacing $T_{k}(\cdot, x) p_{n-k} q_{k} \frac{1}{2^{k}} \sum_{v=1}^{k}\left(\begin{array}{l}k \\ v\end{array}\right)$ by $\frac{1}{R_{m}} \sum_{k=0}^{m} T_{k}(\cdot, x) p_{n-k} q_{k} \frac{1}{2^{k}} \sum_{v=1}^{k}\left(\begin{array}{l}k \\ v\end{array}\right)$ and then by $B_{m}(\cdot, x) p_{n-k} q_{k} \frac{1}{2^{k}} \sum_{v=1}^{k}\left(\begin{array}{l}k \\ v\end{array}\right)$ in (3.1.6) on both sides. For a given $r>0$ choose $\varepsilon^{\prime}>0$ such that $\varepsilon^{\prime}>r$. Define the following sets

$$
\begin{aligned}
& D=\left\{m \leq n:\left\|B_{m}(f, x)-f(x)\right\|_{\infty} \geq r\right\}, \\
& D_{1}=\left\{m \leq n:\left\|B_{m}(1, x)-1\right\|_{\infty} \geq \frac{r-\varepsilon^{\prime}}{4 K}\right\},
\end{aligned}
$$




$$
\begin{aligned}
& D_{2}=\left\{m \leq n:\left\|B_{m}(t, x)-e^{-x}\right\|_{\infty} \geq \frac{r-\varepsilon^{\prime}}{4 K}\right\}, \\
& D_{3}=\left\{m \leq n:\left\|B_{m}\left(t^{2}, x\right)-e^{-2 x}\right\|_{\infty} \geq \frac{r-\varepsilon^{\prime}}{4 K}\right\},
\end{aligned}
$$

Then $\quad D \subset D_{1} \cup D_{2} \cup D_{3}$, and so $\delta(D) \leq \delta\left(D_{1}\right)+\delta\left(D_{2}\right)+\delta\left(D_{3}\right)$.Therefore, using conditions (3.1.1)-(3.1.3), we get

$N E(s t)-\lim _{k \rightarrow \infty}\left\|T_{k}(f ; x)-f(x)\right\|_{\infty}=0 \quad$ This completes the proof of the theorem.

\section{References}

[1] N.Braha, V.Loku and E.Aljimi Weighted Norlund-Euler Statistical Converegnce. Conference proceedings 1st western Balcan Conference of Mathematical sciences Elbasan 1 may 2013.

[2] V. Karakaya, T.A. Chishti, Weighted statistical convergence, Iran. J. Sci. Technol. Trans. A Sci. 33 (2009) 219-223.

[3] N.L. Braha A new class of sequences related to the \$1 $\backslash \mathrm{sb}$ p\$ spaces defined by sequences of Orlicz functions. J. Inequal. Appl. 2011, Art. ID 539745, 10 pp.

[4] Braha, N. L. On asymptotically $\Delta_{\mathrm{m}}$ lacunary statistical equivalent sequences. Appl. Math. Comput. 219 (2012), no. 1, 280-288

[5] Braha, Naim L.; Et, Mikâil. The sequence space $\mathrm{E}_{\mathrm{n}}{ }^{\mathrm{q}}(\mathrm{M}, \mathrm{p}, \mathrm{s})$ and $\mathrm{N}_{\mathrm{k}}$-lacunary statistical convergence. Banach J. Math. Anal. 7 (2013), no. 1, 88-96.

[6] Fast, H. (1951). Sur la convergence statistique. Colloq. Math., 2, 241-244.

[7] Schoenberg, I. J. (1959). The integrability of certain functions and related summability methods. Amer. Math. Monthly, 66, 361-375.

[8] Fridy, J. A. (1985). On statistical convergence. Analysis, 5, 301-313.

[9] Freedman, A. R. \& Sember, I. J. (1981). Densities and summability. Pacific J. Math. 95, 293-305.

[10] Kolk, K. (1991). The statistical convergence in Banach spaces. Acta et Comment. Univ. Tartu., 928, 41-52. 
[11] Kolk, K. (1993). Matrix summability of statistically convergent sequences. Analysis, 13, 77-83.

[12] Fridy, J. A. \& Miller, H. I. (1991). A matrix characterization of statistical convergence. Analysis, 11, 59-66.

[13] Fridy, J. A. \& Orhan, C. (1993). Lacunary statistical convergence. Pacific J. Math., 160, 43-51.

[14] Fridy, J. A. \& Orhan, C. (1993). Lacunary statistical summability. J. Math. Analysis Appl., 173(2), 497-504.

[15] Mursaleen, Mohammad; Karakaya, Vatan; Ertürk, Müzeyyen; Gürsoy, Faik. Weighted statistical convergence and its application to Korovkin type approximation theorem. Appl. Math. Comput. 218 (2012), no. 18, 9132--9137.

[16] Savaş, E. (1992). On strong almost A-summability with respect to a modulus and statistical convergence. Indian J. Pure and Appl. Math. 23(3), 217-222.

[17] Salat, T. (1980). On statistically convergent sequence of real numbers. Math. Slovaca, 30,139-150.

[18] Connor, J. S. (1988). The statistical and strong p-Cesaro convergence of sequence. Analysis, 8, 47-63.

[19] Connor, J. S. (1989). On strong matrix summability with respect to a modulus and statistical convergence.

Canad. Math. Bull., 32, 194-198.

[20] G Hardy, Divergent series, first edition, Oxford University Press, 70. 1949.

[21] G.A. Anastassiou, M. Mursaleen, S.A. Mohiuddine, Some approximation theorems for functions of two variables through almost convergence of double sequences, J. Comput. Anal. Appl. 13 (1) (2011) 37-40.

[22] M. Becker, Global approximation theorems for Szasz-Mirakjan and Baskakov operators in polynomial weight spaces, Indiana Univ. Math. J. 27 (1) (1978) 127-142.

[23] B.D. Boyanov, V.M. Veselinov, A note on the approximation of functions in an infinite interval by linear positive operators, Bull. Math. Soc. Sci. Math.

Roumanie (N.S.) 14 (62) (1970) 9-13. 
[24] O. Duman, K. Demirci, S. Karakus_, Statistical approximation for infinite intervals (preprint).

[25] A.D. Gadjiev, C. Orhan, Some approximation theorems via statistical convergence, Rocky Mountain J. Math. 32 (2002) 129-138.

[26] P.P. Korovkin, Linear Operators and Approximation Theory, Hindustan Publishing Corporation, Delhi, 1960.

[27] S.A. Mohiuddine, An application of almost convergence in approximation theorems, Appl. Math. Lett. 24 (2011) 1856-1860.

[28] F. Moricz, C. Orhan, Tauberian conditions under which statistical convergence follows from statistical summability by weighted means, Studia Sci. Math. Hungar. 41 (2004) 391-403.

[29] M. Mursaleen, A. Alotaibi, Statistical summability and approximation by de la Vallee-Poussin mean, Appl. Math. Lett. 24 (2011) 320-324.

[30] M. Mursaleen, A. Alotaibi, Statistical lacunary summability and a Korovkin type approximation theorem, Ann. Univ. Ferrara 57 (2) (2011) 373-381.

[31] H.M. Srivastava, M. Mursaleen, Asif Khan, Generalized equi-statistical convergence of positive linear operators and associated approximation theorems, Math. Comput. Mod. (2011), doi:10.1016/j.mcm.2011.12.011.

[32] H. Steinhaus, Quality control by sampling, Colloq. Math. 2 (1951) 98-108.

[33] E. Erkus_-Duman, O. Duman, Statistical approximation properties of high order operators constructed with the Chan-Chyan-Srivastava polynomials, Appl. Math. Comput. 218 (5) (2011) 927-1933.

[34] M. Orkcu, O. Dogru, Weighted statistical approximation by kantorovich type q-Szasz-Mirakjan operators, Appl. Math. Comput. 217 (20) (2011) 79137919.

[35] C. Radu, On statistical approximation of a general class of positive linear operators extended in q-calculus, Appl. Math. Comput. 215 (6) (2009) 23172325 .

\section{Received: October 11, 2013}

